Article

\title{
High Frequency Acceleration Device Effect on Accelerated Aligner Exchange - A Pilot Study
}

\author{
Thomas Shipley DMD \\ Adjunct Faculty, Postgraduate Orthodontic Program, \\ Arizona School of Dentistry and Oral Health, A. T. Still University, Mesa, AZ.; \\ Correspondence: thomasshipley@atsu.edu; Tel.: 623-349-7765
}

\begin{abstract}
Evaluation of the effect of a high frequency acceleration device on clear aligner exchange intervals and treatment time required to achieve prescribed tooth movements. Sixteen subjects with similar Class I malocclusions, $5 \mathrm{~mm}$ or less crowding, and treated with Invisalign were divided into two groups. Group 1 (experimental; $\mathrm{N}=8$ ) underwent aligner treatment in conjunction with daily use of the high frequency acceleration device and exchanged aligners every 5 days. Group 2 (controls; $\mathrm{N}=8$ ) underwent aligner treatment without use of the device and exchanged aligners every 14 days according to the manufacturer's recommended interval. All subjects were treated by one investigator, and results were evaluated by both; total number of aligners used, and number of refinements required, prior to final Vivera retention scan. A significant decrease in both treatment time, and number of aligners required to complete treatment was observed by experimental subjects as compared to controls. In addition, no refinements were required by experimental subjects, whereas 6 of 8 of the control subjects required 1 or more refinements. Use of the high frequency acceleration device in conjunction with Invisalign treatment resulted in more predictable tooth movement and a significant decrease in the length of treatment.
\end{abstract}

Keywords: high frequency; acceleration; vibration; orthodontics; tooth movement

\section{Introduction}

Clear aligner therapy has become an increasingly popular orthodontic treatment modality elected by adults and teens alike who seek to avoid traditional fixed braces. However, progression of aligner treatment as well as the estimated time in treatment may not occur as originally planned by the clinician. Even under optimal conditions, where aligner design, treatment planning, and patient cooperation is optimal, the progression of treatment may not completely be expressed clinically according to the original sequentially programmed plan for tooth movement. This may be due to individual variations in physiological and biological factors that impact bone remodeling, and/or external mechanical factors such as patient failure to properly seat aligners or to identify their accurate tracking. Previous literature and studies investigating pulse vibration devices have reported mixed results with regard to their effect on orthodontic treatment. [1-4] While some studies have claimed positive effects on tooth movement, others have reported no effects when compared to controls. Research indicates that that both the vibrational frequency of the device as well as the acceleration (g-force) delivered may play an important role in the capacity for pulse vibration to influence bone remodeling. [5] A recent university based, randomized clinical trial, [1] investigating low-frequency mechanical vibration found no evidence to support accelerated tooth movement or decreased treatment time. Conversely, a recent university based, randomized clinical trial [4] investigating high-frequency mechanical vibration demonstrated both significant accelerated tooth movement and increased cytokine production, a known biological factor in bone remodeling and subsequent orthodontic tooth movement. Independent of the biological factors, accurate physical 
seating of the aligner on all areas of the tooth surface is critical to the accurate and complete transfer of forces to the teeth. [6] Accordingly, a common practice among clinicians has been to recommend the use of treatment adjuncts such as Chewies to facilitate aligner seating. One of the challenges with this practice has been an observable distortion of the aligner. Aligner distortion may result in modified forces applied to the tooth, as well as a significant increase in pain as noted by Fujiyama. [7] Efforts to improve treatment efficiency delivering faster, more predictable tooth movement is at the forefront of modern orthodontic treatment.

HFA facilitates simultaneous maxillary and mandibular full arch, distortion free aligner seating. This in turn delivers optimal, comprehensive, and continuous forces to the teeth. Therefore, it is hypothesized that HFA will support accelerated tray progression and subsequent accelerated tooth movement when compared to traditional orthodontic aligner exchange without the use of HFA. 2.

\section{Materials and Methods}

All subjects gave their informed consent for inclusion before they participated in the study. The study was conducted in accordance with the Declaration of Helsinki, and the protocol was approved prior to commencement by the Ethics Committee of the Chesapeake institutional review board (Pro00020933). The study investigated both the rate of aligner exchange and the time required to complete treatment among subjects that received clear aligner treatment, with and without adjunctive HFA treatment. The inclusion and exclusion criteria are summarized in Table I. All subjects were treated by the same investigator who is certified and experienced in Invisalign clear aligner therapy, and who has completed clear aligner cases with and without adjunctive HFA treatment. All Clincheck treatment plans were prescribed at default aligner velocity. Subjects were consecutively enrolled from treatment records. Per protocol, up to 10 subjects who received clear aligner therapy in conjunction with HFA and an equal number of subjects who received clear aligner therapy without the use of HFA were eligible for this investigation. In total there were 8 completed experimental HFA cases available, all of which met inclusion and exclusion criteria. 10 cases met the protocol criteria for controls, however 2 subjects had also received manual-osteoperforation (MOP) therapy and were therefore excluded.

\section{Table I: Inclusion and exclusion criteria for study participation}

\begin{tabular}{|l|l|}
\hline Inclusion criteria & Exclusion criteria \\
\hline a) Age 14-45 (Male or Female) at initial visit. & a) Subjects with caries present at time of treatment. \\
\hline b) Class I & b) Chronic NSAID, or steroid therapy. \\
\hline c) Crowding mild to moderate (3mm-5mm) & c) Bisphosphonate Therapy. \\
\hline $\begin{array}{l}\text { d) Completed aligner series with Smarttrack } \\
\text { aligner material. }\end{array}$ & d) Pregnancy. \\
\hline e) Post treatment digital Vivera retainer scan. & \\
\hline
\end{tabular}

Treatment data analyzed includes the following: 1. treatment start and finish dates, 2. number of aligners in the initial series, 3. aligner exchange rate in days, 4 . number of case refinements, and 5. any additional aligners required to complete treatment. In order to ensure comparability of selected cases, baseline ABO Discrepancy index [8] (DI) measurements obtained using intra-oral digital 3D measurements and analyzed using OrthoCad ${ }^{\mathrm{TM}}$ [9] version 4.0.4.403 digital model analyzer for each case. All study measurements were performed by the same clinical research associate. To evaluate intrarater measurement reliability, 5 subjects were randomly selected by drawing from study subjects to perform 4 different calibration measures on. Baseline measurements were recorded for overjet, overbite, \#8 incisor width and \#27 canine width, and repeated 1 week and 2 weeks later. Excellent repeatability was demonstrated as measured by Pearson Correlation. Significant correlation for incisor width $(\mathrm{p}=0.05)$. Significant correlation for overjet, overbite and canine width $(\mathrm{p}=0.01)$. 
Statistical Analysis

Categorical variables were compared between groups using the Fisher Exact test. Continuous variables were compared between groups using the t-test for independent samples. Where the parametric assumptions may be questioned, these test results were confirmed by the non-parametric Mann-Whitney $\mathrm{U}$ tests. Paired comparisons within treatment groups were made using paired $\mathrm{t}$-tests. Multiple linear regression was used to simultaneously evaluate the effects of treatment and sex on the number of aligners required. A significance criterion of $p<0.05$ was applied throughout.

\section{Ethical Considerations}

This protocol was submitted and approved by an Institutional Review Board (IRB) prior to study initiation. Data gathered from subject charts was coded to maintain subject confidentiality and privacy.

\section{Results}

The groups were of similar complexity at baseline as measured by the ABO Discrepancy Index, ( $\mathrm{p}=0.536$ by t-test, $\mathrm{p}=0.428$ confirmed by $\mathrm{U}$ test). Table II. Experimental subjects were older than Controls, but not significantly, HFA mean=27.6, SD=11.4, Control mean=18.9, SD=7.8 ( $\mathrm{p}=0.095$ by ttest). All of the HFA subjects were female while the Control group contained 3 females and 5 males ( $p=0.026$ by Fisher Exact test). See Table II for comparative results of primary measures.

\begin{tabular}{|c|c|c|c|}
\hline \multicolumn{4}{|l|}{ Table II. Comparative Results } \\
\hline & Aligner & Aligner + HFA & p value \\
\hline Baseline ABO Discrepancy Index & 16.13 & 12.25 & \multirow{2}{*}{0.536} \\
\hline SD & 14.32 & 9.63 & \\
\hline Baseline Crowding - Upper & $0.95 \mathrm{~mm}$ & $0.74 \mathrm{~mm}$ & \multirow{2}{*}{0.412} \\
\hline SD & $4.45 \mathrm{~mm}$ & $3.47 \mathrm{~mm}$ & \\
\hline Baseline Crowding - Lower & $0.6 \mathrm{~mm}$ & $1.86 \mathrm{~mm}$ & \multirow{2}{*}{0.332} \\
\hline SD & $2.24 \mathrm{~mm}$ & $2.76 \mathrm{~mm}$ & \\
\hline Number of Aligners Prescribed Initially & 29.38 & 25.63 & \multirow{2}{*}{0.224} \\
\hline SD & 6.00 & 5.78 & \\
\hline Estimated Treatment Duration in Weeks & 58.75 & 51.25 & \multirow{2}{*}{0.224} \\
\hline SD & 12.0 & 11.56 & \\
\hline Aligner Exchange Rate Prescribed (days) & 14 & 14 & \multirow{2}{*}{1.0} \\
\hline SD & 0 & 0 & \\
\hline Aligner Exchange Rate Actual (days) & 14 & 4.75 & \multirow{2}{*}{0.001} \\
\hline SD & 0 & 0.70 & \\
\hline Number of Aligners Required to complete & 45 & 25.63 & \multirow{2}{*}{0.001} \\
\hline SD & 10.18 & 5.78 & \\
\hline Actual Treatment Duration in Weeks & 96.75 & 19.25 & \multirow{2}{*}{0.005} \\
\hline SD & 18.76 & 3.88 & \\
\hline Case Refinements & 7 & 0 & \multirow{2}{*}{0.0006} \\
\hline$\%$ & 87.5 & 0 & \\
\hline
\end{tabular}

\subsection{Crowding}

Means for upper crowding did not differ between groups at baseline, $(\mathrm{p}=0.412$ by $\mathrm{t}$-test). Means for lower crowding also did not differ between groups at baseline, $(\mathrm{p}=0.332 \mathrm{by} \mathrm{t}$-test). Both upper and lower crowding were at $0.0 \mathrm{~mm}$ for all subjects in both groups at post-treatment. 


\subsection{Aligner Counts}

The mean number of aligners initially prescribed and actually used are illustrated in Figure 1. The number prescribed was not significantly different between groups, $(\mathrm{p}=0.224$ by $\mathrm{t}$-test), while the number actually used was lower in the HFA group than among Controls, $(\mathrm{p}<0.001$ by $\mathrm{t}$-test).

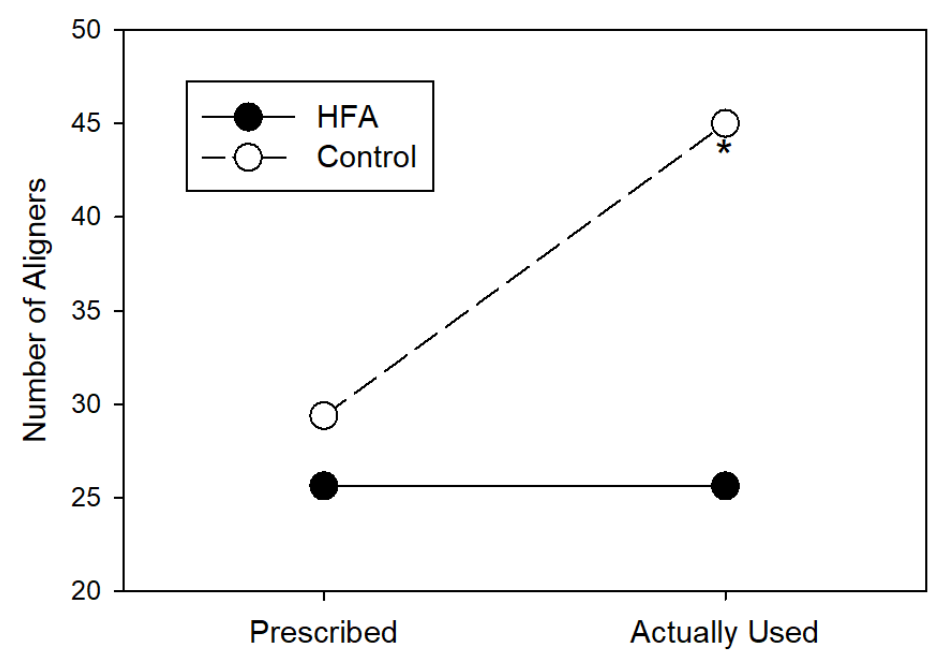

Figure 1. Aligners initially prescribed and actually used.

\subsection{Aligner Change Interval and Treatment Duration}

The prescribed aligner change interval was 14 days for all subjects in both groups. The actual frequency was 14 days for all control subjects. The actual frequency was 5 days for 7 of the HFA subjects and 3 days for 1 HFA subject $(p<0.001$ by $U$ test). The estimated and actual treatment durations are illustrated in Figure 2. The estimated treatment durations did not differ significantly between groups, $(p=0.224$ by t-test). As a result of the aligner counts and change intervals, the treatment duration for HFA was significantly shorter than Control, $(p<0.001$ by t-test and $p<0.001$ confirmed by $U$ test). The HFA group duration was significantly shorter than estimated ( $p<0.001$ by paired $t$-test) while the Control group duration was significantly longer than estimated ( $\mathrm{p}=0.005$ by paired t-test).

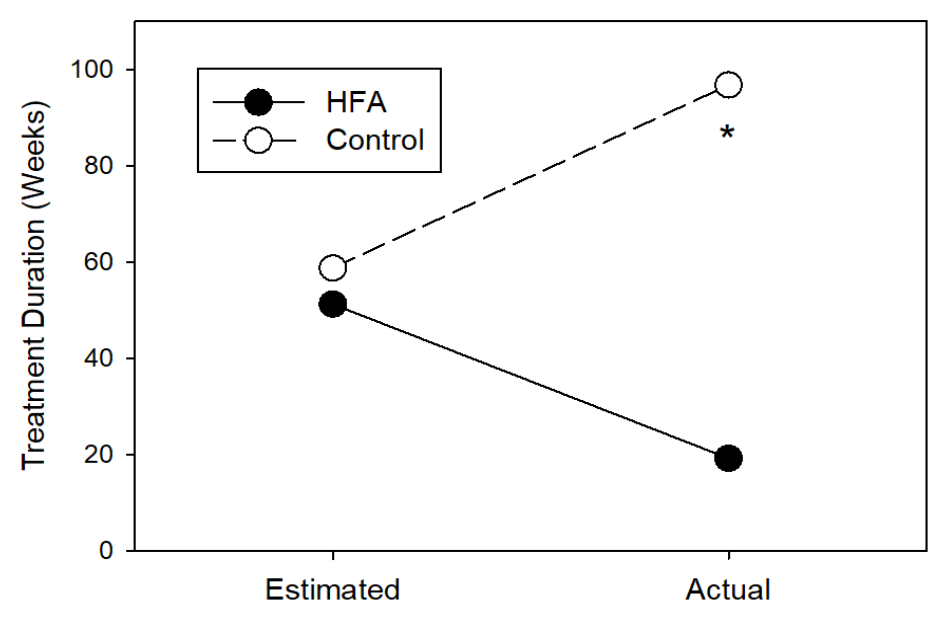

Figure 2. Estimated and actual treatment durations 


\subsection{Case Refinement}

The incidence and number of subjects requiring refinement is illustrated in Figure 3 \& Table II. There were no refinements to the treatment schedule in the HFA group. Six of the 8 Control subjects required refinement ( 5 had 1 refinement, 1 had 2 refinements), adding from 10 to 51 aligner changes $(\mathrm{p}=0.0006$ by paired $\mathrm{t}$-test $)$.

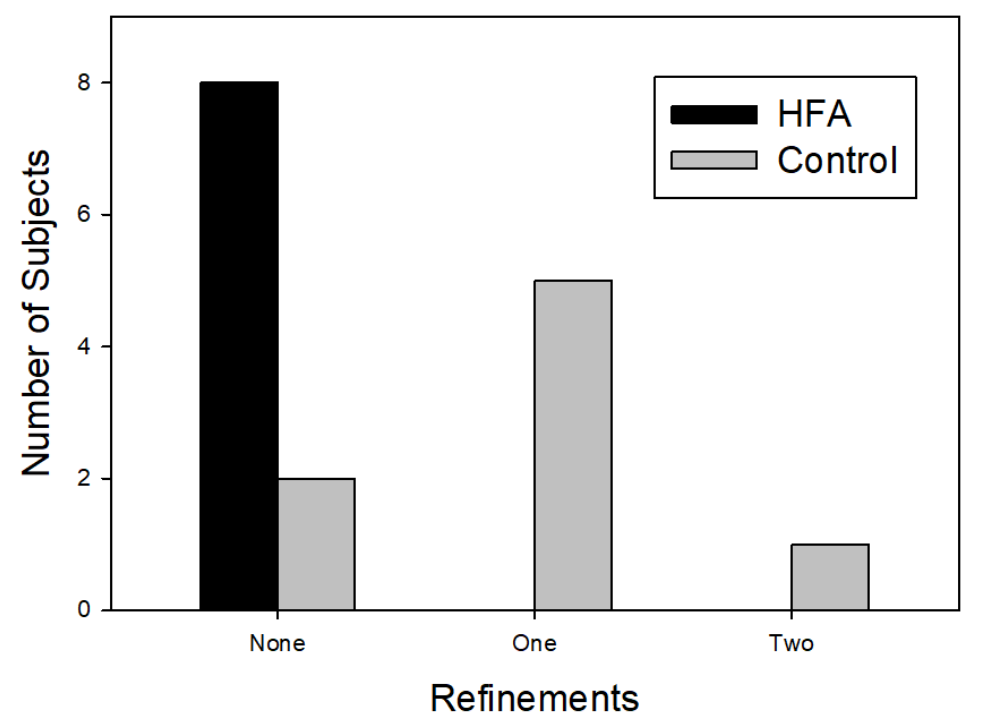

Figure 3. Incidence and number of subjects requiring refinement

\subsection{Potential Sex Bias}

The unequal sex distribution cannot be corrected by statistical methods. However, to test for potential sex effects on aligner changes, multiple regression was employed to test for the influence of treatment and sex on the number of aligners utilized. The regression coefficient for treatment was statistically significant (coefficient=18.71, $\mathrm{SE}=5.81, \mathrm{p}=0.007$ ) while the coefficient for sex was not (coefficient=1.07, SE=6.27, $\mathrm{p}=0.867$ ).

\section{Discussion}

The results of this study demonstrate that clear aligner treatment duration can be significantly reduced using an HFA device by reducing both the intervals between aligner exchanges and the total number of aligners required to complete treatment. Subjects in both groups were diagnosed with statistically similar Class I skeletal malocclusions with mild to moderate crowding, confirmed by baseline ABO Discrepancy Index. Subjects using adjunctive HFA treatment required $43 \%$ fewer aligners to complete their case. While subjects using adjunctive HFA in this study showed no refinements, despite matching initial complexity to controls, this study does not suggest all refinements can be eliminated through incorporating the use of HFA. Limitations of the study include the relatively small sample size but nonetheless demonstrating statistically significant findings with a robust effect size as the first completed cases reported since introduction of the HFA device. This pilot study serves to provide background for effect size calculation on future trials investigating high frequency vibration. The default aligner exchange protocol has subsequently changed from 14-day to a 7-day for select malocclusions at the time of this publication and must be considered in future study design. It is important to note that tray interval is relative to prescription. ClincheckTM treatment planning software at default prescription level delivers a threshold maximum of $0.25 \mathrm{~mm}$ movement allowed per tray. [10] Many clinicians have been using accelerated tray interval 
successfully for years through decreasing the prescribed velocity of tooth movement within their treatment planning software in attempt to improve predictability [11], or reduce pain [12]. However, this practice does not reduce treatment time of initial prescription. For example, a 26-aligner case at default velocity was predicted to span 12 months at the time of this trial. By decreasing prescribed velocity, the same case may be rendered a 52-aligner case spanning the same 12 months at accelerated aligner interval. It is therefore also important to note, all subjects in both groups were at default prescription velocity. Therefore, the HFA Group showed faster more predictable tooth movements, and in this study required no refinements. This finding suggests that extended wear intervals in attempt to improve sequential aligner accuracy may not be as effective as ensuring appliances are consistently fully seated. While office visits were not tabulated in this study, it is assumed that refinement scans, more frequent Clincheck set-ups, and the delivery of additional aligners required to complete treatment resulted in significantly more office visits and doctor time to complete the case for the control group that did not receive HFA treatment. Whether these results may be related to intrinsic biological factors as seen by the increased bone remodeling associated with high frequency vibration in Alikhani's research [2], or extrinsic factors such as optimized force delivery [13] through complete dual arch aligner seating is yet to be fully understood. Future research on these questions is warranted. Consumers and clinicians alike continue to search for more efficient means to straighten teeth. The magnitudes of differences between groups in this preliminary study is striking and consistent with expectations given the background reports.

\section{Conclusions}

1. Accelerated and more predictable tooth movement was achieved using adjunctive HFA treatment with aligner therapy.

2. Use of a HFA device allowed $64 \%$ faster aligner exchanges than control.

3. HFA subjects required fewer refinements than control subjects.

4. HFA subjects required fewer aligners to complete treatment than controls.

Acknowledgments: The high frequency device discussed herein is the commercially available device manufactured by Propel Orthodontics LLC USA.

Conflicts of Interest: The author declares no conflict of interest. The founding sponsors had no role in the design of the study; in the collection, analyses, or interpretation of data; in the writing of the manuscript, or in the decision to publish the results.

\section{References}

1. Woodhouse N. Supplemental Vibrational Force During Orthodontic Alignment: A Randomized Trial., J Dent Res 2015; Apr

2. Alikhani et al. Osteogenic effect of High Frequency on Alveolar Bone. J Dent Res 2012; 91(4):413-419

3. Yadav S, Dobie T, Assefnia A, Gupta H, Kalajzic Z, and Nanda R. Effect of low-frequency mechanical vibration on orthodontic tooth movement. Am J Orthod Dentofac O 2015; 148:440-449

4. Leethanakul C. Vibratory stimulation increases interleukin-1 beta secretion during orthodontic tooth movement. Angle Orthod 2016; Vol 86, No 1

5. Wang L. Effects of Frequency and Acceleration Amplitude on Osteoblast Mechanical Vibration Responses: A Finite Element Study. BioMed Research Intl 2016; Volume 2016, Article ID 2735091

6. Brezniak N. The Clear Plastic Appliance. Angle Orthod 2008; 78(2):381-382.

7. Fujiyama K, Analysis of pain level in cases treated with Invisalign aligner - comparison with fixed edgewise appliance therapy. Progress in Orthodontics 2014; 15:64 
8. ABO Discrepancy Index - A measure of case complexity.

https://www.americanboardortho.com/media/1189/discrepancy index scoring system.pdf

9. OrthoCadTM Digital Model Analyzer for ABO Scoring

http://www.attenborough.com/pdf/OrthoCAD ABO User20Guide.pdf

10. Boyd R. The Invisalign System in Adult Orthodontics - Mild Crowding and Space Closure Cases Journal of Clinical Orthodontics 2000; Vol XXXIV No 4

11. Simon M. Treatment outcome and efficacy of an aligner technique - regarding incisor torque, premolar derotation and molar distalization. BMC Oral Health 2014; 14:68

12. Garrett J. - Effect of reducing the incremental distance of tooth movement per aligner while maintaining the overall rate of movement on self-reported discomfort in Invisalign patients. Masters Thesis, Saint Louis University 2012

13. English J. Mosby's Orthodontic Review. Elsevier Health Sciences 2014; Sep 10, 157-159

(C) 2018 by the authors. Licensee Preprints, Basel, Switzerland. This article is an open access article distributed under the terms and conditions of the Creative Commons by Attribution (CC-BY) license (http://creativecommons.org/licenses/by/4.0/). 